\title{
THE EFFICACY OF COMBINED BIOMARKER TESTS IN EARLY DIAGNOSTICS OF ACUTE MYOCARDIAL INFARCTION AFTER CLINICAL SYMPTOMS ONSET: ROLE OF HEART-TYPE FATTY ACID-BINDING PROTEIN
}

\author{
O.V. Baturina, S.R. Gilyarevsky*, I.M. Kuzmina, M.A. Godkov, E.V. Klychnikova \\ Research Institute of Emergency Medicine named after N.V. Sklifosovsky \\ Bolshaya Sukharevskaya pl. 3, Moscow, 129090, Russia
}

The efficacy of combined biomarker tests in early diagnostics of acute myocardial infarction after clinical symptoms onset: role of heart-type fatty acid-binding protein
O.V. Baturina, S.R. Gilyarevsky*, I.M. Kuzmina, M.A. Godkov, E.V. Klychnikova
Research Institute of Emergency Medicine named after N.V. Sklifosovsky. Bolshaya Sukharevskaya pl. 3, Moscow, 129090, Russia

Aim. To evaluate the diagnostic value of heart-type fatty acid-binding protein (H-FABP) introduction to standard cardiac biomarkers in diagnostics of myocardial infarction (MI) early after clinical symptoms onset.

Material and methods. Patients aged 18 years and older admitted to the hospital with acute coronary syndrome (ACS) during first 6 hours after ACS symptoms onset were enrolled into the Material and methods. Patients aged 18 years and older admitted to the hospital with acute coronary syndrome (ACS) during first 6 hours after ACS symptoms onset were enrolled into the
study. At hospitalization level of biomarkers of myocardial damage (creatine phosphokinase-MB fraction - CPK-MB, troponin T) and qualitative tests on FABP were assessed in all patients. Serum troponin T concentration measured in 12 hours after the hospitalization was used to confirm diagnosis of MI. Moreover, diagnosis of MI was verified by echocardiography with the assessment of local reduction in myocardial contractility.

Results. 101 patients were included into the study ( $27 \%$ female; aged $59.9 \pm 12.4$ ). At the moment of hospitalization 71 (70\%) patients were diagnosed with ACS with ST-segment elevation, and $30(30 \%)$ patients - ACS without ST-segment elevation. In total, period between clinical symptoms onset and hospitalization was 3.6 \pm 1.4 hours.

Analysis of diagnostic value of tests during the first 6 hours after ACS symptoms onset showed higher sensitivity of H-FABP in comparison with troponin T and CPK-MB ( $72.5,56.2$ and $49.5 \%$ Analysis of diagnostic value of tests during the first 6 hours after ACS symptoms onset showed higher sensitivity of H-FABP in compariso
respectively). Specificity of these tests during the first 6 hours after onset of clinical signs of ACS was 90,100 n $90 \%$, respectively.

Sensitivity and specificity were 87.7 and $100.0 \%$, respectively, when H-FABP, troponin T and CPK-MB were used in combination as a "block test" in patients hospitalized during the first 6 hours after ACS symptoms onset. Sensitivity and specificity were 74.3 и 100.0\%, respectively, when "block test" was used in patients hospitalized during the first 3 hours after ACS symptoms onset; between 3 and 6 hours after ACS symptoms onset sensitivity and specificity of tests reached $100 \%$.

Conclusion. "Block test" with H-FABP, troponin T and CPK-MB early after ACS symptoms onset is more sensitive in comparison with separate use of the tests.

Key words: heart-type fatty acid-binding protein, acute coronary syndrome, "block" test.

Rational Pharmacother. Card. 2012;8(3):405-414

\begin{abstract}
Эффективность сочетанного применения тестов на биомаркеры для диагностики инфаркта миокарда в ранние сроки после развития его клинических проявлений: роль кардиоспецифического белка, связывающего жирные кислоты

О.В. Батурина, С.Р. Гиляревский*, И.М. Кузьмина, М.А. Годков, Е.В. Клычникова

Научно-исследовательский институт скорой помощи им. Н.В. СКлифосовского. 129090, Москва, Большая Сухаревская пл., д. 3

Цель. Оценить диагностическую эффективность добавления кардиоспецифического белка, связывающего жирные кислоты (ксБСжК), к стандартным кардиоспецифическим биомаркерам для диагностики инфаркта миокарда (ИМ) в ранние сроки после развития клинических проявлений заболевания.

Материал и методы. В исследование включали пациентов в возрасте 18 лет и старше, госпитализированных по поводу острого коронарного синдрома (ОКС) в первые 6 ч от развития клинических проявлений заболевания.

Всем больным в момент госпитализации проводили оценку уровня кардиоспецифических маркеров повреждения миокарда (МВ фракция креатинфосфокиназы - МВ-КФК, тропонин Т) и качественные тесты на БСЖК. Через 12 ч после госпитализации повторно определяли концентрацию тропонина Т в крови. Результаты количественного анализа крови на тропонин Т через 12 ч после госпитализации использовали для подтверждения диагноза ИМ. Кроме того, во всех случаях диагноз ИМ подтверждали результатами эхокардиографии с оценкой нарушения локальной сократимости миокарда.

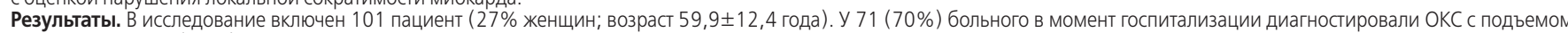
сегмента ST, а у 30 (30\%) больных - ОКС без подъема сегмента ST. Продолжительность периода между развитием симптомов заболевания и госпитализацией в целом достигала $3,6 \pm 1,44$

Результаты анализа диагностической эффективности тестов в течение первых 6 ч после развития клинических проявлений ОКс свидетельствовали о более высокой чувствительРезультаты анализа диагностической эффективности тестов в течение первых 6 ч после развития клинических проявлений ОКС свидетельствовали о более высокой чувствитель-
ности ксБСЖК по сравнению с чувствительностью тропонина Т и МВ-КФК (чувствительность таких тестов составляла 72,5; 56,2 и 49,5\%, соответственно). Специфичность указанных тестов в течение первых 6 ч после развития клинических проявлений ОКС достигала 90, 100 и 90\%, соответственно.

При сочетанном применении тестов на ксБСЖК, тропонин Т и МВ-КФК в качестве «блокового теста» у больных, госпитализированных в течение первых 6 ч от развития ОКС, чувствительность и специфичность достигала 87,7 и 100,0\%, соответственно. При использовании «блокового теста» у больных, госпитализированных в первые 3 ч от развития ОКС, чувствительность и специфичность составляла 74,3 и 100,0\%, соответственно, а в период между 3 и 6 ч от развития ОКС - чувствительность и специфичность достигали 100\%. Заключение. Применение «блокового теста» с использованием ксБСЖК, тропонина Т и МВ-КФК в ранние сроки после развития клинических проявлений ОКС, имеет более высокую чувствительность по сравнению с применением каждого из тестов в отдельности.

Ключевые слова: кардиоспецифический белок, связывающий жирные кислоты, острый коронарный синдром, «блоковый» тест.

РФК 2012;8(3):405-414
\end{abstract}

*Corresponding author (автор, ответственный за переписку): sgilarevsky@rambler.ru

\section{Authors' information:}

Oxana V. Baturina - MD, Doctor of Cardiology Department No. 3 for patients with myocardial infarction, Research Institute of Emergency

Medicine named after N. V. Sklifosovsky

Sergey R. Gilyarevsky - MD, PhD, Professor, Head of the same

Department

Irina M. Kuzmina - MD, PhD, Leading Researcher of the same Department Mikhail A. Godkov - MD, PhD, Professor, Head of Laboratory Diagnostics Department, Research Institute of Emergency Medicine named after N.V. Sklifosovsky

Elena V. Klychnikova - MD, PhD, Interim Head of Laboratory of Clinical biochemistry of urgent tests, Research Institute of Emergency Medicine named after N.V. Sklifosovsky
Сведения об авторах:

Батурина Оксана Владимировна - врач 3-го кардиологического отделения для больных инфарктом миокарда НИИ СП им Н.В. СКлифосовского

Гиляревский Сергей Руджерович - Д.м.Н., профессор, руководитель того же отделения

Кузьмина Ирина Михайловна - К.М.Н., В.Н.С. того же отделения Годков Михаил Андреевич - д.м.н., профессор, руководитель отдела лабораторной диагностики НИИ СП им Н.В. СКлифосовского Клычникова Елена Валерьевна - К.м.Н., и. о. руководителя клинико-биохимической лаборатории экстренных методов исследования НИИ СП им Н.В. Склифосовского 
Myocardial infarction (MI) is one of the most common causes of death and disability [1]. In prevalence studies, WHO defined MI by clinical symptoms, pathological electrocardiogram (ECG) abnormalities and enzymes levels. However identification of more sensitive and specific serological biomarkers, as well as more precise visualization methods, allowed to detect myocardial necrosis of smaller sizes. Therefore, a more precise MI diagnostics is required in current clinical practice and in epidemiological and clinical studies.

Also it should be taken into consideration that accuracy of Ml diagnostics will change with introduction of more specific biomarkers of myocardial necrosis. Such changes took place when aspartate aminotransferase, glutamic-oxaloacetic transaminase were replaced by lactate dehydrogenase, and then by creatine phosphokinase (CPK) and its $\mathrm{MB}$ fraction (CPK-MB), i.e. activity of CPK-MB and its mass. Nowadays the usage of more sensitive biomarkers and visualization methods represent a new step in this evolution.

Timeliness of MI diagnosis is essential because it generally determines initiation of treatment and volume of intervention. In accordance with current Guidelines [2] diagnosis of $\mathrm{Ml}$ is based on analysis of patients complaints and anamnestic data, as well as ECG abnormalities and elevation of at least one of the biomarkers of myocardial necrosis - first of all troponin I or T. The most significant limitation in troponin use is its low sensitivity for MI diagnostics in very early terms after symptoms onset: elevation of troponin concentration is defined in 6-8 hours depending on Ml size. Therefore, Ml can be not recognized during first hours after symptoms onset because anamnestic data, patients complaints, ECG results and tests for standard biomarkers often remain undetermined at this period. [3, 4].

Recent studies have showed the efficacy of hearttype fatty acid-binding protein (H-FABP) as an early biomarker of myocardial damage for MI diagnostics, as well as its significance in defining prognosis of patients after MI. [5-8]. Furthermore, experts of both European Society of Cardiology and National Academy of Clinical Biochemistry, USA [9] offer combined usage of several biomarkers for MI diagnostics. The results of the first clinical studies allow to suggest that this approach is more effective for Ml diagnostics in different terms after symptoms onset including the earliest ones [10]. Recently in Russia several studies have been performed to assess the efficacy of H-FABP test for early MI diagnostics $[11,12]$. However, according to our data, there are no studies, evaluating the effi-
Инфаркт миокарда (ИМ) относится к наиболее частым причинам смерти и инвалидности [1]. В исследованиях по оценке распространенности ИМ, эксперты Всемирной организации здравоохранения определяли ИМ на основании клинических симптомов, патологических изменений электрокардиограммы (ЭКГ) и уровня ферментов. Однако открытие более чувствительных и специфичных серологических биомаркеров, а также создание более точных методов визуализации позволило выявлять некрозы миокарда все меньшего размера. Следовательно, в ходе реализации существующей клинической практики, а также в ходе проведения эпидемиологических и клинических исследований требуется более точная диагностика ИМ.

Следует также принять во внимание, что с появлением доступных более специфичных биомаркеров некроза миокарда, изменится и точность выявления ИМ. Такие изменения произошли, когда вместо аспартатаминотрансферазы, глутаминоксалоацетаттрансаминазы стали применять лактатдегидрогеназу, а затем креатинфосфокиназу (КФК) и ее MB-фракцию (МВ-КФК), т.е. активность МВ-КФК и массу МВ-КФК. В настоящее время использование более чувствительных биомаркеров и методов визуализации представляют новый этап в этой эволюции.

Своевременность установления диагноза ИМ представляется принципиальной, поскольку ранняя диагностика, как правило, обуславливает соответствующие сроки начала лечения и объем применяемых вмешательств. В соответствии с современными рекомендациями [2] диагноз ИМ устанавливают на основании анализа жалоб больного и данных анамнеза, а также изменений ЭКГ и повышения концентрации хотя бы одного биомаркера повреждения миокарда, и, прежде всего, тропонинов I или Т.

К наиболее существенным ограничениям в использовании тропонина относят его низкую чувствительность для диагностики ИМ в очень ранние сроки после развития клинических проявлений заболевания: в зависимости от размера ИМ повышение концентрации тропонина в крови выявляется через 6-8 ч после развития симптомов. Следовательно, в течение первых часов после развития симптомов ИМ может быть не распознан, поскольку данные анамнеза, а также жалобы больного, результаты ЭКГ и тестов на стандартные биомаркеры в этот период нередко остаются недостаточно определенными [3, 4].

Результаты ранее выполненных исследований свидетельствовали об эффективности применения кардиоспецифического белка, связывающего жирные кислоты (ксБСЖК) в качестве раннего биомаркера повреждения миокарда для диагностики ИМ, а также о значении этого маркера для определения прогноза больных, перенесших ИМ [5-8]. Более того, эксперты как Европейского общества кардиологов, так и Национальной академии клинической биохимии США [9] предлагают сочетанное использование нескольких биомаркеров для диагностики ИМ. Результаты первых клинических испытаний позволяют предположить, 
cacy of combined use of biomarkers, including $\mathrm{H}$-FABP test, for MI diagnostics in early period after symptoms onset.

Aim of the study: To evaluate the diagnostic efficacy of heart-type fatty acid-binding protein (H-FABP) introduction to standard cardiac biomarkers in diagnostics of Ml in early period after clinical symptoms onset.

\section{Material and methods}

Patients with suspected ACS hospitalized to the intensive care unit of the cardiology department No. 3 for patients with Ml of Research Institute of Emergency Medicine named after N.V. Sklifosovsky from February 2011 to January 2012 were enrolled into the study.

Inclusion criteria: 1) age 18 years and older; 2) duration of chest pain which caused hospitalization 20 min or more; 3) duration of period between symptoms onset and hospitalization less than 6 hours.

Exclusion criteria: history of chronic diseases which are able to cause persistent elevation of the cardiac biomarkers (particularly, chronic heart failure and chronic renal insufficiency).

Depending on the duration of the period between ACS symptoms onset and hospitalization, the patients were split in two groups: Group 1 - duration is less than 3 hours; Group 2 - duration is from 3 to 6 hours.

At the moment of hospitalization venous blood samples of all patients were collected for biochemical analysis, estimation of myocardial damage (CPK MB, troponin $T$ ) markers and for qualitative H-FABP tests. In 12 hours after hospitalization blood samples were taken repeatedly for the evaluation of troponin T concentration. Quantitative test for troponin T was used to confirm the diagnosis of MI. Besides, in all cases diagnosis of MI was verified by echocardiography with the assessment of local reduction in myocardial contractility.

Qualitative H-FABP test was used in this study (express test "CardioBSZhK", NPO Biotest, Novosibirsk, Russia). H-FABP test technique was the following: $150 \mu \mathrm{l}$ of heparinized blood is put in the plate's window; tests results were assessed in 15$20 \mathrm{~min}$. If the test strip was colored the test was considered as "positive". Two independent experts evaluated the test results. The test was assessed as positive only if experts opinions coincided. Serum troponin $\mathrm{T}$ concentration was measured using quantitative electrochemiluminescence method on automatic analyzer "Elecsys 2010" (Roche, Germany). что такой подход более эффективен для диагностики ИМ в разные сроки после развития клинических проявлений заболевания, включая самые ранние [10]. В последнее время в России выполнено несколько исследований по оценке эффективности применения теста на ксБСЖК для ранней диагностики ИМ $[11,12]$. Однако по нашим данным, отсутствуют работы, в которых специально изучалась эффективность сочетанного применения биомаркеров, включая тест на ксБСЖК, для диагностики ИМ в ранние сроки после развития его клинических проявлений.

Цель исследования: оценить диагностическую эффективность добавления ксБСЖК к стандартным кардиоспецифическим биомаркерам для диагностики ИМ в ранние сроки после развития клинических проявлений заболевания.

\section{Материал и методы}

В исследование были включены больные, которые в период с февраля 2011 г. по январь 2012 г. были госпитализированы в палату реанимации и интенсивной терапии 3-го кардиологического отделения для больных ИМ НИИ скорой помощи им. Н.В. Склифосовского с предполагаемым диагнозом острого коронарного синдрома (ОКС).

Критерии включения: 1) возраст 18 лет и более; 2) продолжительность приступа болей в грудной клетке, которые стали причиной госпитализации, 20 мин или более; 3) продолжительность периода между развитием клинических проявлений заболевания и госпитализацией не более 64 .

Критерии исключения: наличие в анамнезе хронических заболеваний, которые могут обуславливать стойкое повышение уровня кардиоспецифических биомаркеров (в частности, хронической сердечной недостаточности и хронической почечной недостаточности).

В зависимости от продолжительности периода между развитием клинических проявления ОКС и госпитализацией, больные были распределены на две группы: группа 1 - продолжительность менее 3 ч; группа 2 - продолжительность в диапазоне 3-6 ч.

Всем больным в момент госпитализации брали кровь из вены как для биохимического анализа крови и оценки концентрации кардиоспецифических маркеров повреждения миокарда (МВ-КФК, тропонин Т), так и для выполнения качественных тестов на БСЖК. Через 12 ч после госпитализации повторно брали кровь для определения концентрации тропонина Т в крови. Результаты количественного анализа крови на тропонин Т через 12 ч после госпитализации использовали для подтверждения диагноза ИМ. Кроме того, во всех случаях диагноз ИМ подтверждался результатами эхокардиографии с оценкой нарушения локальной сократимости миокарда.

В ходе исследования использовали качественный тест на БСЖК (экспресс-тест «КардиоБСЖК», НПО Биотест, Новосибирск). Методика выполнения теста на БСЖК была следующей: 150 мкл гепаринизированной крови вносили в окно 


\section{Statistical analysis}

Continuous data were represented as mean \pm standard deviation, and qualitative data were compared using $\chi^{2}$ test. In all cases differences were considered significant at $p<0.05$.

Sensitivity and specificity were calculated using formulas: sensitivity $=a /(a+c)$, specificity $=d /(b+d)$, where $a$ - true positive results, $b$ - false positive results, $c$ - false negative results, $d$ - true negative results.

In addition, a prognostic value of the test was calculated (probability of presence/absence of disease at known result of the study).

A prognostic value of the true positive result (PVPR), i.e. probability of presence of disease at positive (pathological) result, was calculated using a formula: PVPR $=a /(a+b)$, where $a-$ true positive results, $b-$ false positive results.

A prognostic value of the true negative result (PVNR), i.e. probability of absence of disease at negative (non-pathological) result was calculated using a formula: $P V N R=c /(c+d)$, where $c$ - false negative results, $d$ - true negative results.

An explorative analysis was also planned in the subgroup of patients with ACS without ST-segment elevation on ECG (nonST-ACS).

Statistical analysis was performed using MedCalc 11.0 software package (MedCalc Software, Belgium) and MS Excel 2007 (Microsoft, USA).

\section{Results}

101 patients were enrolled into the study ( $27 \%$ female; mean age $59.9 \pm 12.4$ ). At the moment of hospitalization ACS with ST-segment elevation was diagnosed in 71 (70\%) patients, and ACS without ST-segment elevation - in 30 (30\%) patients. In total, period between symptoms onset and hospitalization was $3.6 \pm 1.4$ hours.

The first group consisted of 44 patients (27\% female), the second one - 57 patients (36\% female).

Clinical and demographic characteristics of patients enrolled into the study are presented in the table 1.

In subgroup of non-ST segment elevation ACS MI was confirmed in 21 patients, and unstable angina - in 9 patients.

Thrombolytic therapy (TLT) was performed in 16 patients, including 14 patients with prehospital TLT (effective one in 8 cases). In two cases of in-hospital TLT was effective; percutaneous coronary intervention $(\mathrm{PCl})$ with stent placement was performed in 69 (68\%) patients.

In general, analysis of diagnostic efficacy of tests планшеты, результат теста оценивался через 15-20 мин. При наличии окрашивания тестовой полоски тест расценивали как положительный. Результаты теста оценивали два независимых эксперта. Тест считали положительным только при совпадении мнения экспертов. Концентрацию тропонина Т в крови определяли с использованием количественного электрохемилюминесцентного анализа на автоматическом анализаторе «Elecsys 2010» (Roche, Германия).

\section{Методы статистического анализа}

Непрерывные данные представляли как среднее значение \pm стандартное отклонение, а качественные признаки сравнивали с помощью критерия $\chi^{2}$. Во всех случаях различия считали статистически значимыми при уровне $p<0,05$.

Чувствительность и специфичность рассчитывали по формулам: чувствительность $=a /(a+c)$, специфичность $=d /(b+d)$, где a - истинно положительные результаты, $\mathrm{b}$ - ложноположительные результаты, с - ложноотрицательные результаты, $\mathrm{d}$ - истинно отрицательные результаты.

Кроме того, рассчитывали прогностическую ценность теста (вероятность наличия/отсутствия заболевания при известном результате исследования).

Прогностическую ценность положительного результата (ПЦПР), т.е. вероятность наличия заболевания при положительном (патологическом) результате теста, рассчитывали по формуле: ПЦПР=а/ $(\mathrm{a}+\mathrm{b})$, где $\mathrm{a}-$ истинно положительные результаты, $b$ - ложноположительные результаты.

Прогностическую ценность отрицательного результата (ПЦОР), т.е. вероятность отсутствия заболевания при отрицательном (непатологическом) результате теста, рассчитывали по формуле: ПЦОР=c/(c+d), где c - ложноотрицательные результаты, $d$ - истинно отрицательные результаты.

Был также запланирован поисковый анализ в подгруппе больных с ОКС без подъема сегмента ST на ЭКГ (ОКСБПST).

Статистический анализ полученных результатов выполняли с помощью пакета программ MedCalc 11.0 (MedCalc Software, Бельгия) и MS Excel 2007 (Microsoft, США).

\section{Результаты}

В исследование включен 101 пациент (27\% женщин; средний возраст 59,9 12,4 года). У 71 (70\%) больного в момент госпитализации диагностировали ОКС с подъемом сегмента ST и лишь у 30 (30\%) больных - ОКСБПSТ. Продолжительность периода между развитием симптомов заболевания и госпитализацией в целом достигало 3,6士1,4 4.

Первую группу составили 44 пациента (27\% женщин), вторую - 57 пациентов (36\% женщин).

Клинико-демографическая характеристика больных, включенных в исследование, представлена в табл. 1.

Диагноз ИМ в подгруппе ОКСБПST ходе обследования был установлен у 21 больного, и у 9 больных этой подгруппы диагностировалась нестабильная стенокардия.

Тромболитическую терапию (ТЛТ) выполнили у 16 больных. В 14 случаях ТЛТ применяли на догоспитальном этапе (у 8 
Table 1. Participants characteristics

Таблица 1. Характеристики больных, включенных в исследование

\begin{tabular}{|c|c|c|c|}
\hline Parameter / Параметр & $\begin{array}{l}\text { All patients / Все больные } \\
\qquad(n=101)\end{array}$ & $\begin{array}{c}\text { Group } 1 \text { / Группа } 1 \\
(n=44)\end{array}$ & $\begin{array}{c}\text { Group } 2 \text { / Группа } 2 \\
(n=57)\end{array}$ \\
\hline Male / Мужчины, \% & 73 & 73 & 64 \\
\hline Age, years / Возраст, лет & $59.9 \pm 12.4$ & $60.0 \pm 12.6$ & $59.9 \pm 12.4$ \\
\hline \multicolumn{4}{|c|}{ Diseases and risk factors in the past medical history / Заболевания или факторы риска в анамнезе } \\
\hline Arterial hypertension / Артериальная гипертония, n (\%) & $94(93)$ & $42(95)$ & $52(91)$ \\
\hline Myocardial infarction / Инфаркт миокарда, n (\%) & $19(19)$ & $13(30)$ & $6(11)$ \\
\hline Diabetes mellitus / Сахарный диабет, n (\%) & $18(18)$ & $9(20)$ & $9(16)$ \\
\hline Smoking / Курение, n (\%) & $39(39)$ & $18(41)$ & (21) 37 \\
\hline \multicolumn{4}{|c|}{ Type of acute coronary syndrome / Тип острого коронарного синдрома } \\
\hline With ST elevation / С подъемом сегмента ST, n (\%) & $71(70)$ & $32(72)$ & $39(68)$ \\
\hline Non-ST elevation / Без подъема сегмента ST, n (\%) & $30(30)$ & $12(27)$ & $18(32)$ \\
\hline Coronarography performance / Выполнение коронарографии, n (\%) & $93(92)$ & $44(100)$ & $49(86)$ \\
\hline PCl performance / Выполнение ЧКВ, n (\%) & $69(68)$ & $33(75)$ & $35(61)$ \\
\hline
\end{tabular}

during first 6 hours after ACS symptoms onset showed higher sensitivity of $\mathrm{H}-\mathrm{FABP}$ in comparison with troponin $\mathrm{T}$ and $\mathrm{CPK}-\mathrm{MB}(72.5,56.2$ and $49.5 \%$, respectively). Specificity of these tests during first 6 hours after ACS symptoms onset was 90,100 and $90 \%$, respectively (figure). Diagnostic efficacy of tests in the Group 1 also indicated

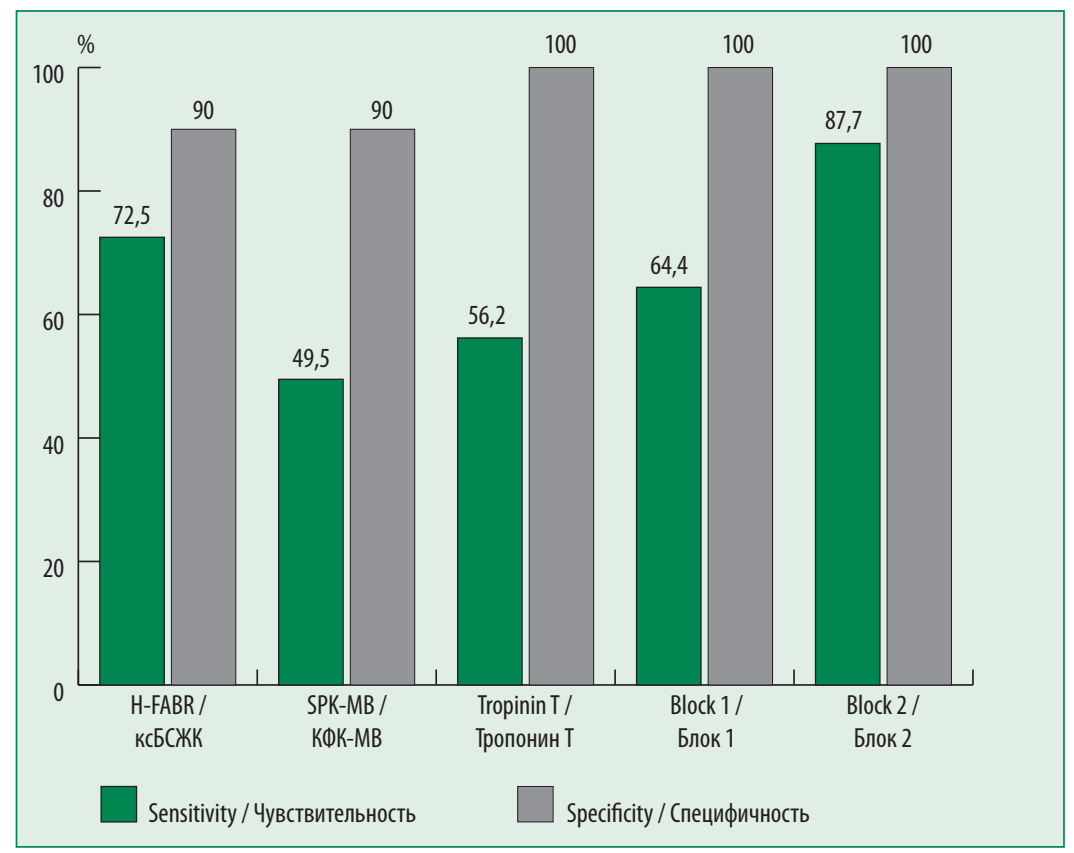

Figure. Assessment of sensitivity and specificity of biomarkers of myocardial damage and their combined use in blocks

Block 1 included a combined evaluation of concentration of CPK-MB and troponin T, and block 2 - CPK-MB, troponin Т и H-FABR

Рисунок. Результаты оценки чувствительности и специфичности биомаркеров повреждения миокарда, а также их сочетанного использования в виде блоков

Блок 1 включал сочетанную оценку концентрации КФК-МВ и тропонина Т, блок 2 - КФК-МВ, тропонина Т и ксБСЖК больных она была эффективной). В обоих случаях применения ТЛТ в стационаре она была эффективной; чрескожное коронарное вмешательство (ЧКВ) со стентированием выполнили у $69(68 \%)$ больных.

В целом результаты анализа диагностической эффективности тестов в течение первых 6 ч после развития клинических проявлений ОКС свидетельствовали о более высокой чувствительности ксБСЖК по сравнению с чувстви тельностью тропонина Т и МВ-КФК (чувствительность таких тестов составляла 72,5; 56,2 и 49,5\% соответственно). Специфичность указанных тестов в течение первых 64 после развития клинических проявлений OKC достигала 90, 100 и 90\% соответственно (рис.).

Результаты анализа диагностической эффективности тестов в группе 1 также указывали на более высокую чувствительность ксБСЖК по сравнению с чувствительностью тропонина Т и МВ-КФК (чувствительность тестов в такие сроки после развития клинических проявлений ОКС достигала 54,8, 31,4 и 26,2\%, соответственно). Специфичность всех тестов в группе 1 достигала 100\% (табл. 2)

Чувствительность ксБСЖК, тропонина Т и МВ-КФК в группе 2 составляла 87,8, 79,0 и $69,4 \%$, соответственно, а специфичность 87,$5 ; 100,0$ и 87,5\%, соответственно.

При сочетанном использовании тестов на тропонин Т и МВ-КФК у больных, госпитализированных в целом в течение первых 6 ч после развития клинических проявлений OKC, чувствительность и специфичность 
Table 2. Assessment of sensitivity and specificity of biomarkers of myocardial damage

Таблица 2. Результаты оценки чувствительности и специфичности биомаркеров повреждения миокарда

\begin{tabular}{|c|c|c|c|c|c|}
\hline Value / Показатель & $\begin{array}{c}\text { H-FАВР / } \\
\text { ксБСЖК }\end{array}$ & $\begin{array}{l}\text { CPK-MB / } \\
\text { КФК-MB }\end{array}$ & $\begin{array}{l}\text { Troponin T / } \\
\text { Тропонин T }\end{array}$ & $\begin{array}{c}\text { Block (CPK-MB + } \\
\text { Troponin T) / } \\
\text { Блок (КФК-MВ + } \\
\text { Тропонин Т) }\end{array}$ & $\begin{array}{c}\text { Block (CPK-MB + } \\
\text { Troponin T + H-FABP) / } \\
\text { Блок (КФК-МВ + } \\
\text { Тропонин Т + кСБСЖК) }\end{array}$ \\
\hline \multicolumn{6}{|c|}{ Group 1 (<3 hours) / Группа 1 (<3ч) } \\
\hline Sensitivity / Чувствительность, \% & 54.8 & 26.2 & 31.4 & 42.9 & 74.3 \\
\hline Specificity / Специфичность, \% & 100 & 100 & 100 & 100 & 100 \\
\hline PVPR / ПЦПР, \% & 100 & 100 & 100 & 100 & 100 \\
\hline PVNR / ПЦОР, \% & 90.5 & 93.9 & 92.3 & 90.9 & 81.8 \\
\hline \multicolumn{6}{|c|}{ Group 2 (3-6 hours) / Группа 2 (3-6 ч) } \\
\hline Sensitivity / Чувствительность, \% & 87.8 & 69.4 & 79.0 & 84.2 & 100 \\
\hline Specificity / Специфичность, \% & 87.5 & 87.5 & 100 & 100 & 100 \\
\hline PVPR / ПЦПР, \% & 97.7 & 97.1 & 100 & 100 & 100 \\
\hline PVNR / ПЦОР, \% & 46.2 & 68.2 & 50 & 81.8 & 0 \\
\hline \multicolumn{6}{|c|}{ Total* / В целом* } \\
\hline Sensitivity / Чувствительность, \% & 72.5 & 49.5 & 56.2 & 64.4 & 87.7 \\
\hline Specificity / Специфичность, \% & 90 & 90 & 100 & 100 & 100 \\
\hline PVPR / ПЦПР, \% & 98.5 & 97.8 & 100 & 100 & 100 \\
\hline PVNR / ПЦОР, \% & 73.5 & 83.6 & 76.2 & 72.2 & 47.4 \\
\hline \multicolumn{6}{|c|}{$\begin{array}{l}\text { *all patients hospitalized during first } 6 \text { hours after ACS symptoms onset; H-FABP = heart-type fatty acid-binding protein; CPK-MB = creatine phosphokinase-MB fraction; PVPR = } \\
\text { prognostic value of the true positive result; PVNR = prognostic value of the true negative result } \\
\text { *у всех больных, госпитализированных в течение первых } 6 \text { ч после развития клинических проявлений ОКС коронарного синдрома; ксБСЖК = кардиоспецифический } \\
\text { белок, связывающий жирные кислоты; МВ-КФК = МВ-фракция креатинфосфокиназы; ПЦПР = прогностическая ценность положительного результата; ПцОТ = прогности- } \\
\text { ческая ценность отрицательного результата }\end{array}$} \\
\hline
\end{tabular}

higher sensitivity of $\mathrm{H}$-FABP than of troponin $\mathrm{T}$ and CPK-MB (sensitivity of these tests early after ACS symptoms onset was $54.8,31.4$ and $26.2 \%$, respectively). Specificity of all tests in the Group 1 was 100\% (table 2).

Sensitivity of H-FABP, troponin T and CPK-MB in the Group 2 was 87.8, 79.0 and $69.4 \%$, respectively, and specificity was $87.5,100.0$ and $87.5 \%$, respectively.

When troponin $\mathrm{T}$ and $\mathrm{CPK}-\mathrm{MB}$ tests were combined in patients hospitalized during the first 6 hours after ACS symptoms onset, sensitivity and specificity were 64.4 and $100 \%$, respectively. When these tests were used in combination in patients of Group 1, sensitivity and specificity were 42.9 n $100.0 \%$, respectively, and in patients of Group 2 sensitivity increased up to $84.2 \%$, while specificity remained $100 \%$.

Sensitivity and specificity were 87.7 and $100.0 \%$, respectively, when $\mathrm{H}-\mathrm{FABP}$, troponin $\mathrm{T}$ and $\mathrm{CPK}-\mathrm{MB}$ tests were used in combination as a "block test" in patients hospitalized during the first 6 hours after ACS symptoms onset. Sensitivity and specificity was 74.3 и $100.0 \%$, respectively, when "block test" was used in the Group 1; sensitivity and specificity in the Group 2 reached 100\%.

An exploratory analysis was performed in the достигала 64,4 и 100,0\%, соответственно. При сочетанном использовании комбинации этих тестов у больных группы 1 чувствительность и специфичность составляла 42,9\% и 100,0\%, соответственно, а в группе 2 чувствительность увеличивалась до $84,2 \%$ при сохранении специфичности $100 \%$.

При сочетанном применении тестов на ксБСЖК, тропонин Т и МВ-КФК в качестве «блокового теста» у больных, госпитализированных в целом в течение первых 6 ч после развития клинических проявлений ОКС, чувствительность и специфичность достигала 87,7 и 100,0\%, соответственно. При использовании такого "блокового теста» у больных группы 1 чувствительность и специфичность составляла 74,3 и 100,0\%, соответственно, а в группе 2 чувствительность и специфичность достигали $100 \%$.

Был также выполнен поисковый анализ в подгруппе больных с OKСБПST ( $n=30)$ для подтверждения эффективности использования изучаемой тактики применения биомаркеров для ранней диагностики ИМ. В целом результаты анализа диагностической эффективности тестов в течение первых 6 ч после развития клинических проявлений ОКС в этой подгруппе также свидетельствовали о более высокой чувствительности ксБСЖК по сравнению с чувствительностью тропонина Т и МВ-КФК (чувствительность таких тестов составляла $65,0,62,5$ и 55,0\%, соответственно), хотя по сравнению со всей группой больных чувствительность ксБСЖК была меньше (72,5 и $65,0 \%$, соответственно). Специфичность указанных тестов в 
subgroup of non-ST segment elevation ACS $(n=30)$ to confirm the efficacy of the studied tactics of biomarkers usage for the early MI diagnostics. In total, analysis of the tests diagnostic efficacy during the first 6 hours in this subgroup also showed higher sensitivity of $\mathrm{H}$ - FABP than this of troponin $\mathrm{T}$ and CPK-MB (65.0, 62.5 и 55.0\%, respectively), though sensitivity of $\mathrm{H}$-FABP was less than that in the whole group ( 72.5 and $65.0 \%$, respectively). Specificity of all these tests during the first 6 hours after ACS symptoms onset was 90, 100 and 90\%, respectively, and was consistent with patients data with any ACS type. However sensitivity of H-FABP test during the first 3 hours after ACS symptoms onset was less than that of troponin $\mathrm{T}$ ( 44.4 and $50.0 \%$, respectively). At the same time, between 3 and 6 hours after ACS symptoms onset the sensitivity of $\mathrm{H}$-FABP test became higher again than that of troponin $\mathrm{T}$ ( 81.8 and $72.7 \%$, respectively). When used as a "block test" (H-FABP, CPK-MB and troponin T) during the first 3 hours after ACS symptoms onset, the test sensitivity reached $93.8 \%$, while specificity remained $100 \%$. Thereby, analysis in the subgroup of non-ST segment elevation ACS during the first 3 hours after ACS symptoms onset showed high efficacy of the approach using "block test" for MI diagnostics in patients hospitalized with suggested diagnosis of non-ST segment elevation ACS. Sensitivity and specificity of "block test" for MI diagnostics between 3 and 6 hours after symptoms onset reached $100 \%$.

\section{Discussion}

Timeliness of ACS diagnostics in patients right after hospitalization to the intensive care unit is essential because it determines initiation of optimal therapeutic strategy. Moreover, an exclusion of Ml is important in preventing unreasonable examination and hospital stay.

Despite the presence of several diagnostic markers of myocardial damage, including troponins and CPK-MB, that characterized by rather high specificity, the role of such biomarkers in early MI diagnostics is substantively limited by their low sensitivity during the first 6 hours after disease symptoms onset.

At the same time a rather large observational study [13] showed that a less intensive therapy is used for patients with first Ml if they have a negative troponin test results during the first hours after disease symptoms onset, and that probability of reinfarction in such patients is twofold higher during 6 months of follow-up. It should be noted that first MI with negative serum troponin test is rather течение первых 6 ч после развития клинических проявлений ОКС достигала 90\%, 100\% и 90\%, соответственно, что совпадало с результатами анализа данных о больных с любым типом ОКС. Однако чувствительность теста на ксБСЖК в первые З ч после развития симптомов ОКС оказалась меньше, чем таковая для тропонина Т (44,4 и 50,0\%, соответственно). В то же время в период между 3 и 6 ч после развития симптомов ОКС чувствительность теста на ксБСЖК вновь становилась выше, чем таковая тропонина Т ( $81,8 \%$ и 72,7\% соответственно). При использовании «блокового теста» (ксБСЖК, КФК-МВ и тропонин Т) у больных в первые 3 ч после развитии симптомов ОКС чувствительность теста достигала 93,8\% при сохранении специфичности в 100\%. Таким образом, результаты анализа в подгруппе больных с ОКСБПSТ в первые 3 часа после развития симптомов ОКС подтвердили данные о высокой эффективности применения такого «блокового» подхода к диагностике ИМ и у больных, госпитализированных с предположительным диагнозом OKСБПST. Чувствительность и специфичность применения «блокового теста» к диагностике ИМ в период от 3 до 6 ч после развития клинических проявлений заболевания достигала $100 \%$.

\section{Обсуждение}

Своевременное установление точного диагноза ОКС у больных в ранние сроки после госпитализации в отделение интенсивной терапии относят к важным условиям применения оптимальной тактики лечения. Более того, представляется важным и возможность исключения диагноза ИМ, что позволяет избежать необоснованного обследования и пребывания больного в стационаре.

Несмотря на то, что имеется несколько диагностических маркеров повреждения миокарда, включая тропонины и MB-КФК, которые характеризуются достаточно высокой специфичностью, низкая чувствительность таких биомаркеров в течение первых 6 ч после развития клинических проявлений заболевания существенно ограничивает их роль в ранней диагностике ИМ.

В то же время в ходе выполнения достаточно крупного обсервационного исследования [13] были получены данные о том, что у больных с первым ИМ при отрицательном тесте на тропонин в крови в течение первых часов после развития клинических проявлений заболевания применяется менее активная тактика лечения, и у них в два раза выше вероятность развития повторного ИМ в течение 6 мес наблюдения. Следует отметить, что первый ИМ с отрицательным тестом на тропонин в крови встречается достаточно часто, а тактика лечения больных с таким инфарктом должна быть более активной.

Для преодоления указанных ограничений для ранней диагностики ИМ с помощью биомаркеров предлагается несколько подходов. В частности, в последнем варианте клинических рекомендаций по диагностике и лечению больных с OKCБПST [14] для усовершенствования диагностики ИМ в ранние сроки после развития клинических проявлений предлагается выполнение серии тестов на высокочувствительный тропонин, 
common, and therapeutic strategy for patients with such infarctions should be more active.

There are several approaches to overcome above limitations of early MI diagnostics using biomarkers. Particularly, the latest clinical guidelines for diagnostics and treatment of patients with non STsegment elevation ACS [14] suggest to use tests series for high sensitive troponin performed at first at hospitalization and then in 3 hours to improve early MI diagnostics after symptoms onset. If at least one value exceeds 99th percentile of normal range of the biomarker concentration, the MI diagnosis is considered to be confirmed. T. Keller et al. study [15] showed that the use of both high sensitive and standard troponin I tests provides rather high PVPR (for exclusion of MI) during the first 3 hours after hospitalization of the patient, which was 75.1 and $80.9 \%$, respectively. In spite of the fact that recommendations for use of such algorithm corresponds to the class I [14], such algorithm is not included in the American guidelines for the management of patients with nonST-ACS [16], and validity of such test is to be proved in further studies. Anyway it's reasonable to continue searching for optimal approaches to early MI diagnostics using biomarkers of myocardial damage, including H-FABP.

Our results suggest higher sensitivity of $\mathrm{H}$-FABP test in comparison with troponin $\mathrm{T}$ and CPK-MB ones for MI diagnostics in patients hospitalized during the first 6 hours after ACS symptoms onset. Sensitivity of H-FABP, troponin T and CPK-MB tests in such terms after symptoms onset was $72.5,56.2$ and $49.5 \%$, respectively. Our data concerning rather high sensitivity of $\mathrm{H}$-FABP between 3 and 6 hours after ACS onset, which was $87.8 \%$, in general consist with studies of C.G. McMahon et al. [10] and Figiel Ł. et al. [17], where this value was 85.3 and $87.0 \%$, respectively. However, according to both our data and C.G. McMahon et al. [10], sensitivity of H-FABP during the first 3 hours after symptoms onset was not high enough (54.8 and $64.3 \%$, respectively).

Altogether, high sensitivity of $\mathrm{H}$-FABP test can be explained by its high level in myocardium in comparison with other tissues. When used as a biomarker of myocardial damage, H-FABP also has such advantages as it's stability and good solubility, low molecular weight ( $15 \mathrm{kDa}$ ) and early release into blood circulation (already in $60 \mathrm{~min}$ ) after episode of myocardial ischemia [18], as well as relatively high tissue specificity [19].

Results of this study, suggesting that during the first 6 hours after disease symptoms onset specificity of $\mathrm{H}$-FABP test is lower than of troponin T test сначала - в момент госпитализации больного, а затем - через 3 ч. Если хотя бы одно значение превышает 99\% процентиль нормальных значений концентрации этого биомаркера, диагноз ИМ считается подтвержденным. Результаты исследования T. Keller и соавт. [15] свидетельствовали о том, что при использовании теста как на высокочувствительный, так и на стандартный тропонин I, такой алгоритм диагностики обеспечивает достаточно высокую ПЦПР (для исключения ИМ) в течение первых 3 ч после госпитализации больного, которая достигала 75, 1 и $80,9 \%$, соответственно. Несмотря на то, что рекомендации по использованию такого алгоритма применения теста на тропонин для ранней диагностики ИМ соответствуют I классу [14], такой алгоритм пока не включен в американские рекомендации по ведению больных с ОКСБПSТ [16], а обоснованность его использования еще предстоит подтвердить в ходе выполнения дополнительных исследований. Как бы там ни было, представляется целесообразным продолжить поиск оптимальных подходов к ранней диагностике ИМ с помощью биомаркеров повреждения миокарда, в том числе и с использованием кСБСЖК.

Полученные нами данные свидетельствуют о более высокой чувствительности использования теста скБСЖК по сравнению с оценкой концентрации тропонина Т и МВ-КФК для диагностики ИМ у больных, госпитализированных в течение первых 6 ч после развития клинических проявлений ОКС. Чувствительность скБСЖК, тропонина Т и МВ-КФК в такие сроки после развития симптомов достигала 72,$5 ; 56,2$ и 49, $5 \%$, соответственно. Полученные нами данные о достаточно высокой чувствительности ксБСЖК в период от 3 до 6 ч после развития клинических проявлений ОКС (составляла 87,8\%), в целом совпадают с результатами исследований C.G. McMahon и соавт. [10] и Figiel Ł. и соавт. [17], в ходе которых этот показатель достигал 85,3и 87,0\%, соответственно. Однако, как по нашим данным, так и по данным C.G. McMahon и соавт. [10], чувствительность ксБСЖК в первые 3 ч после развития клинических проявлений была недостаточно высокой, составляя 54,8 и 64,3\%, соответственно.

В целом высокую чувствительность теста на ксБСЖК объясняют высоким уровнем ксБСЖК в миокарде по сравнению с другими тканями. К преимуществам ксБСЖК при использовании в качестве биомаркера повреждения миокарда относят также его стабильность и хорошую растворимость, низкую молекулярную массу ( 15 кДа) и высвобождение в кровь в ранние сроки (уже через 60 мин) после эпизода ишемии миокарда [18], а также относительно высокую тканевую специфичность [19].

Полученные в данном исследовании результаты, свидетельствующие о том, что в течение первых 6 ч после развития симптомов заболевания, специфичность теста на ксБСЖК несколько ниже, чем теста на тропонин Т (90 и 100\%, соответственно) в целом совпадают с результатами исследования C.G. McMahon и соавт. [10]. Сочетанное применение тропонина Т и МВ-КФК для диагностики ИМ существенно повышало не только чувствительность теста в целом, но и его специфичность, которая, как при исследовании в течение первых 3 4, так и в 
(90 and 100\%, respectively), generally consist with the results of C.G. McMahon et al. study [10]. Combined use of troponin T and CPK-MB for MI diagnostics significantly increased not only sensitivity of the test in general, but also its specificity which was $100 \%$ both during first 3 hours and between 3 and 6 hours after MI symptoms onset.

At the same time it should be noted that not all of the studies supported high diagnostic value of the combined $\mathrm{H}$-FABP and troponin I test in patients with suggested MI. Particularly, results of Freund $\mathrm{Y}$. et al. study [20], which included 317 ACS patients hospitalized to the emergency department, showed that PVNR of the combined H-FABP and troponin I test $(96 \%$ at $95 \%$ confidence interval [Cl] $93-$ 98\%) did not exceed PVNR of the troponin I test alone ( $95 \%$ at $95 \% \mathrm{Cl} 93-98 \%$ ).

\section{Study limitations}

In spite of the fact that our study confirms validity of the "block" approach to the MI diagnostics using the tests for biomarkers of myocardial damage, some limitations should be noted. Although the most important role of the tests for biomarkers is in the Ml diagnostics in patients hospitalized with nonST-ACS, only $30 \%$ of patients had such type of ACS in our study. Nevertheless, analysis in the nonST-ACS subgroup confirmed a high diagnostic value of the "block" approach to the $\mathrm{Ml}$ diagnostics very early after $\mathrm{MI}$ symptoms onset (less than 3 hours) and in such type of ACS. For confirmation of the results it is appropriate to conduct an additional study including more participants with both ST-segment elevation ACS and nonST-ACS, to provide sufficiently high statistical power, including analyses in subgroups with different types of ACS.

Also it should be noted that inclusion of more patients with ST-segment elevation MI allowed better evaluation of specificity of the assessed diagnostic methods of myocardial damage. Anyway, the received data confirm high diagnostic value of $\mathrm{H}$-FABP and, particularly, combined biomarkers test using H-FABP.

\section{Conclusion}

So "block" approach to the tests for biomarkers of myocardial damage using $\mathrm{H}$-FABP, troponin $T$ and CPK-MB early after ACS symptoms onset is more sensitive than each of above tests alone.

Disclosures. All authors have not reported about potential conflicts of interest for this article. период между 3 и 6 ч после развития симптомов ИМ, достигала $100 \%$.

В то же время следует отметить, что результаты не всех исследований подтверждали высокую диагностическую ценность сочетанного использования теста на ксБСЖК и тропонина I у больных с предполагаемым ИМ. В частности, результаты исследования Freund Y. и соавт. [20], в которое были включены 317 больных с ОКС, доставленных в отделение неотложной помощи, свидетельствовали о том, что ПЦОР теста для сочетанного теста на ксБСЖК и тропонин I не превышала таковой при изолированном применении теста на тропонин I, достигая 96\% при 95\% доверительном интервале (95\%ДИ) 93-98\% при сочетанном применение двух тестов, и 95\% при 95\%ДИ 93-98\% - при изолированном применении теста на тропонин I.

\section{Ограничения исследования}

Результаты выполненного нами исследования в целом подтверждают обоснованность использования «блокового» подхода к диагностике ИМ с помощью тестов на биомаркеры повреждения миокарда, при этом следует отметить ряд недостатков данного исследования. Несмотря на то, что наибольшую роль тесты на биомаркеры играют в диагностике ИМ у больных, госпитализированных с ОКСБПSТ, в нашем исследовании только 30\% больных имели такой тип ОКС. Тем не менее, результаты анализа в подгруппе больных с ОКСБПSТ подтвердили высокую диагностическую ценность «блокового» подхода к диагностике ИМ в очень ранние сроки после развития его клинических проявлений (менее 3 ч) и при таком типе ОКС. Для подтверждения результатов анализа целесообразно выполнение дополнительного исследования с включением большего числа больных как с OKC с подъемом сегмента ST, так и OKCБПST, что обеспечивало бы достаточно высокую статистическую мощность анализа, в то числе анализа в подгруппах больных с разным типом OKC.

Следует также отметить, что включение в исследования большого числа больных с ИМ с подъемом сегмента ST позволяло более надежно оценить специфичность оцениваемых методов диагностики повреждения миокарда. В любом случае, полученные данные подтверждают высокую диагностическую ценность ксБСЖК, и особенно, сочетанного применения биомаркеров с использованием ксБСЖК.

\section{Заключение}

Таким образом, применение «блокового» подхода к использованию тестов на биомаркеры повреждения миокарда с использованием ксБСЖК, тропонина Т и МВ-КФК в ранние сроки после развития клинических проявления ОКС имеет более высокую чувствительность по сравнению с применением каждого из тестов в отдельности.

Конфликт интересов. Авторы не сообщили об отсутствии потенциального конфликта интересов по данной статье. 


\section{References / Литература}

1. Roger V.L., Go A.S., Lloyd-Jones D.M., et al. Heart disease and stroke statistics - 2012 update: a report from the American Heart Association. Circulation 2012; 125: e2-e220.

2. Thygesen K., Alpert J.S., White H.D., et al. Universal definition of myocardial infarction. Circulation 2007; 116: 2634-2653.

3. Thygesen K., Alpert J.S., White H.D. Universal definition of myocardial infarction. J Am Coll Cardiol 2007; 50: $2173-2195$

4. Bassand JP, Hamm CW, Ardissino D, et al. Guidelines for the diagnosis and treatment of non-ST-segment elevation acute coronary syndromes. Eur Heart J 2007; 28: 1598-1660.

5. Glatz J., van der Vusse G., Simoons M., et al. Fatty acid-binding protein and the early detection of acute myocardial infarction. Clinica Chimica Acta 1998; 272: 87-92

6. O'Donoghue M., deLemos J., Morrow D., et al. Prognostic utility of heart-type FABP in patients with acute coronary syndromes. Circulation 2006;114: 550-557.

7. Tanaka T., Hirota Y., Sohmiya K., et al. Serum and urinary human h-FABP in acute myocardial infarction. Clin Biochem 1991; 24: 195-201.

8. Kilcullen N., Viswanathan K., Das R., et al. Heart-type fatty acid binding protein predicts long-term mortality after acute coronary syndrome and identifies high-risk patients across the range of troponin values. J Am College Cardiology 2007; 50: 2061-2067.

9. Morrow D.A., Cannon C.P., Jesse R.L., et al. National Academy of Clinical Biochemistry Laboratory Medicine Practice Guidelines: clinical characteristics and utilization of biochemical markers in acute coronary syndromes. Clin Chem 2007; 53: 552-574.

10. McMahon C.G., Lamont J.V., Curtin E., et al. Diagnostic accuracy of heart-type fatty acid-binding protein for the early diagnosis of acute myocardial infarction. Am J Emerg Med 2012; 30: 267-274.

11. Golovenkin S.E., Shul'man V.A., Pelipetskaya E.Yu., et al. The use of rapid test "KardioBSZhK" for the earIy diagnosis of myocardial necrosis in patients with acute coronary syndrome. Spravochnik Vracha Obshchey Praktiki 2012; (1); 29-40. Russian (Головенкин С.Е., Шульман В.А., Пелипецкая Е.Ю., и др. Применение экспресс-теста «КардиоБСЖК» для ранней диагностики некроза миокарда у больных острым коронарным синдромом. Справочник Врача Общей Практики 2012; (1); 29-40).

12. Ryabov V.V., Suslova T.E., Markov V.A. i dr. Determination of protein and other fatty acid binding, in the diagnosis of acute myocardial infarction. Byulleten SO RAMN 2005; (3): 26-29. Russian (Pябов В.В., Суслова Т.Е., Марков В.А. и др. Определение белка, связывающего жирные кислоты, в диагностике острого инфаркта миокарда. Бюллетень СО РАМН 2005; (3): 26-29).
13. Corteville D.C., Armstrong D.F., Montgomery D.G., et al. Treatment and outcomes of first troponinnegative non-ST-segment elevation myocardial infarction. Am J Cardiol 2011; 107: 24-29.

14. Hamm C.W., Bassand J.P., Agewall S., et al. ESC Guidelines for the management of acute coronary syndromes in patients presenting without persistent ST-segment elevation: The Task Force for the management of acute coronary syndromes (ACS) in patients presenting without persistent ST-segment elevation of the European Society of Cardiology (ESC). European Heart Journal 2011; 32: 2999-3054.

15. Keller T., Zeller T., Ojeda F., et al. Serial Changes in Highly Sensitive Troponin I Assay and Early Diagnosis of Myocardial Infarction. JAMA 2011; 306: 2684-2693.

16. Wright R.S., Anderson J.L., Adams C.D., et al. 2011 ACCF/AHA focused update incorporated into the ACC/AHA 2007 Guidelines for the Management of Patients with Unstable Angina/Non-ST-Elevation Myocardial Infarction: a report of the American College of Cardiology Foundation/American Heart Association Task Force on Practice Guidelines developed in collaboration with the American Academy of Family Physicians, Society for Cardiovascular Angiography and Interventions, and the Society of Thoracic Surgeons. J Am CollCardiol 2011; 57: e215-367.

17. Figiel $Ł$., Wraga M., Bednarkiewicz Z., et al. Direct comparison of the diagnostic value of point-ofcare tests detecting heart-type fatty acid binding protein or glycogen phosphorylaseisoenzyme BB in patients with acute coronary syndromes with persistent ST-segment elevation. Kardiol Pol 2011;69: $1-6$.

18. Van Nieuwenhoven F.A., Kleine A.H., Wodzig W.H., et al. Discrimination between myocardial and skeletal muscle injury by assessment of the plasma ratio of myoglobin over fatty acid binding protein. Circulation 1995; 92: 2848-54.

19. Alhadi H.A., Fox K.A. Do we need additional markers of myocyte necrosis: the potential value of heart fatty-acid-binding protein? QJM 2004; 97: 187-198.

20. Freund Y., Chenevier-Gobeaux C., Leumani F., et al. Heart-type fatty acid binding protein and the diagnosis of acute coronary syndrome in the ED. Am J Emerg Med 2011. [Epub ahead of print].

Received/Поступила 27.03.2012 Accepted/Принята в печать 10.05.2012 